\title{
Novel Approach for Evaluating Video Transmission using Combined Scalable Video Coding over Wireless Broadband Network
}

\author{
Kalvein Rantelobo ${ }^{1}$, Kalamullah Ramli ${ }^{2}$, Wirawan ${ }^{3}$ \\ ${ }^{1}$ Department of Electrical Engineering, Universitas Nusa Cendana, Indonesia \\ ${ }^{2}$ Department of Electrical Engineering, Universitas Indonesia, Indonesia \\ ${ }^{3}$ Department of Electrical Engineering, Institut Teknologi Sepuluh Nopember, Indonesia
}

\begin{tabular}{l}
\hline \hline Article Info \\
\hline Article history: \\
Received Oct 18, 2017 \\
Revised May 21, 2018 \\
Accepted May 28, 2018 \\
\hline
\end{tabular}

\section{Keyword:}

CSVC

MGS

NS-2

Video transmission

Wireless broadband network

\begin{abstract}
One of the main problems in video transmission is the bandwidth fluctuation in wireless channel. Therefore, there is an urgent need to find an efficient bandwidth utilization and method. This research utilizes the Combined Scalable Video Coding (CSVC) which comes from Joint Scalable Video Model (JSVM). In the combined scalable video coding, we implement Coarse Grain Scalability (CGS) and Medium Grain Scalability (MGS). We propose a new scheme in which it can be implemented on Network Simulator II (NS-2) over wireless broadband network. The advantages of this new scheme over the other schemes are more realistic and based on open source program. The result shows that CSVC implementation on MGS mode outperforms CGS mode.
\end{abstract}

Copyright @ 2018 Institute of Advanced Engineering and Science. All rights reserved.

Corresponding Author:

Kalvein Rantelobo,

Departement of Electrical Engineering,

Universitas Nusa Cendana,

Jl. Adisucipto, Penfui, Kupang, NTT, Indonesia.

Email: rantelobo@ieee.org

\section{INTRODUCTION}

An increasing use of technology and the advanced development of information technology require a large capacity of bandwidth and mobile services. These lead to the badwidth fluctuation particularly those that use video transmission. Furthermore, wireless channel condition and bandwidth availability determine the quality of video transmission. One of the attractive methods to solve the aforementioned problems in video transmission over wireless channel is layering transmission method. In this method, bit streams are scaled into a number of layer which consists of base and enhancement layers.. This is known as scalable video method. This method is also implemented in multicast network where receivers only receive video transmission as needed [1].

A systematic review of the vision of scalable video coding (SVC) over broadband is given in [1]. Moreover the application of the performances of SVC and its applications is shown in [2]-[4]. SVC is known in three basics which are quality scalable (SNR scalable), temporal scalable, and spatial scalable. The combination of these basics, known as Combined Scalable Video Coding (CSVC), is carried out by combining the advantage of each one to transmit video when bit rate is prioritized and SNR scalable is used when quality is preferred. The advantage of CSVC lies in the solution that it provides for video transmission from various inputs in heterogeneous networks with multiple terminals having different capacities.

Most research used SVC with JSVM but not the combination of different scalable methods. This will affect the quality of end to end system. The fore, in this research, we use the combination of SVC (combined scalable video coding). This will be more flexible for adaptation to input characters, fluctuation of 
channel transmission, and multicast on network conditions. There are several researchers that proposed the use of SCVS such as hybrid temporal-FGS (fine granular scalability) by Mihaela [5] and spatio-temporal by Blaszak [6]. In this research, we extend the CSVC method proposed by Schwarz [7]. The current application of SVC on broadband services has been proposed by Maodong Li [8]. There is a limited number of reported works on evaluation of video transmission in wireless broadband networks. Thus far the research community in the performance of evaluating video transmission on the network uses Evalvid [9] as popular scheme and tools. Evalvid limitation which only supports MPEG standard has encouraged the scheme, new methods and tools developed and refined by various parties. In [10], the proposed myEvalvid is seen to be capable to run H.264, but have not been yet able to run SVC.

There are several schemes that utilize SVC as a public evaluation platform and based on opensource program. They are, SVEF (Scalable Video coding Evaluation Framework) [11], EvalSVC (Evaluation of SVC) [12], and myEvalSVC [13]. In [11], the proposed SVEF schemes and tools are built on open-source basis. SVEF is a mixed online/offline open-source framework devised to evaluate the performance of H.264 SVC video streaming. SVEF is written in C and Python and also will be released under the GNU General Public License. SVEF has been able to evaluate basic SVC. However, it is still incomplete and inadequate in analyzing complete SVC. In addition, SVEF construction may not easy since it requires specialized skills. EvalSVC method and scheme proposed in [12] are publicly available and free. However, EvalSVC does not clearly indicate how to handle missing or corrupted packets. It is then updated and extended in [13] with myEvalSVC which is capable to run H.264 AVC and SVC in general. Nevertheless, this scheme has not been explored and developed to the maximum so that the package has not been able to evaluate the CSVC. To overcome those limitations, we propose a new scheme in this paper. The scheme provides a tool-set to evaluate and analyze CSVC in a wireless broadband network on an end-to-end system.

This scheme is developed and distributed on an open-source platform to enable researcher and developer to use, analyze, and enrich it according to their requirements and needs [14]. To obtain the comprehensive analysis, the combination of scalable method is needed. However, to the best of our knowledge, there is no research on evaluation SVC using the combination of scalable method, which is a key in evaluation the quality of video transmission over broadband network. In this research, we compare the MGS and CGS, which is an extension of our previous [15], [16].

The scheme of framework can evaluate performance of average Peak Signal-to- Noise Ratio (PSNR), coding efficiency, performance of metrics systems as end-to-end delay, and queuing delay of the packets in the network. Furthermore, the scheme is simpler than other schemes in prior work which used to evaluate of video transmission over wireless broadband network (it will be explained in more detail in the next section of this paper). The proposed scheme is a promising approach for evaluating video transmission over wireless broadband services in the future. We believe that this new scheme will facilitate and simplify the evaluation and exploitation of video packets over wireless broadband network which is expected to improve research in this field.

The rest of the paper is organized as follows: Section 2 presents the CSVC evaluation framework, CSVC method, MGS and CGS modes, and Network Simulator over wireless broadband network. In Section 3, we describe the experimental results and discussion, and finally we present conclusion and future work.

\section{THE COMBINED SCALABLE VIDEO CODING (CSVC) EVALUATION FRAMEWORK 2.1. Combined scalable video coding (CSVC)}

SVC is a part of H.264/MPEG-4 part 10 AVC (Advanced Video Coding), or H.264/AVC [1]-[3]. H.262 and MPEG-2, followed by H.263+ and MPEG-4. Since January 2005, MPEG and VCEG have been joined together in JVT to carry out completion of the H.264/AVC amendment as an official standard [2]. Until now, H.264/AVC standard is still in amendment, and it is a cooperative work of many parties to establish Multiview Video Coding and High Efficiency Video Coding (HEVC) or H.265.

Scalability proposed for the first time is to reduce packet (cell) loss in ATM networks [1]. It divides one bitstream into several sub-bitstreams or layers according to network state, which can offer efficiency and superior quality video coding on broadband services compared to other video coding standards. It created two groups of layers which are base and enhancement layers. The base layer contains vital information whereas the enhancement layer comprises residual information to enhance the quality of video sequences. A SVC system consists of encoder and decoder is depicted in Figure 1. Within data transmission process, base-layer containing vital information still makes it through in case of congestion in transmission channel. Figure 1 shows the three types of scalability methods: SNR (Signal-to-Noise Ratio) Scalability, Spatial Scalability, and Temporal Scalability. In addition, there is the other scalability method that combines these three methods. When the inter layer resolution is changed, the spatial scalable will be dominant. When the quality 
of video is changed, SNR scalable is dominant. When the bit rate is changed, the temporal scalability is dominant. The combination of those three is likely to due to varying in sequences characteristics, fluctuated network condition and multi terminals [1], [5], [7]. This research utilizes three layers of combined scalable: one base layer and two enhancement layers as given in Figure 1.

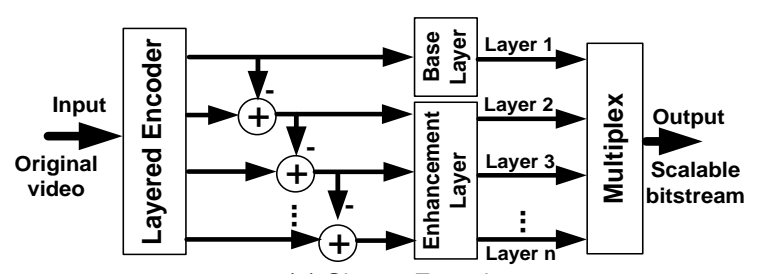

(a) Sistem Encoder

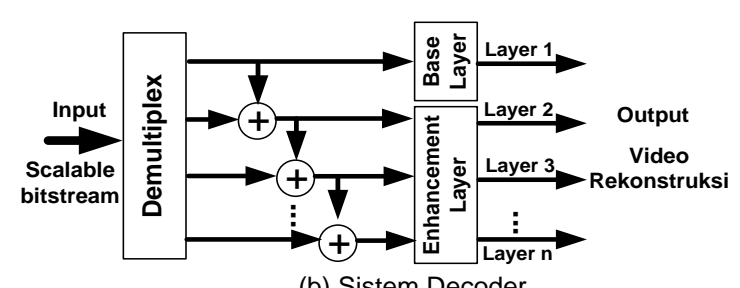

(b) Sistem Decoder

Figure 1. Scalable video coding systems

\subsection{MGS and CGS}

In this work, we focus on the quality scalability in H.264 SVC. Layer of quality scalable has the same spatio-temporal resolution but different fidelity. The H.264 SVC extension supports two quality scalable modes namely coarse grain scalability (CGS) and medium grain scalability (MGS) [2], [17]. Each spatial layer is referred to as CGS or MGS. CGS can be viewed as a special case of spatial scalability in H.264 SVC. In similar encoding mechanisms, CGS is employed but the spatial resolution will remain constant. More specifically, similar to spatial scalability, CGS employs inter-layer prediction mechanisms, such as prediction of macro block modes and associated motion parameters and prediction of the residue signal. CGS differs from spatial scalability where up-sampling operations are not performed. In CGS, the residual texture signal in the enhancement layer is re-quantized with a smaller quantization step size than the preceding CGS layer. SVC supports up to eight CGS layers fit to eight quality extraction points, namely one base layer and up to seven enhancement layers. If quality scalability is using CGS, switching different CGS layers must be done at the defined point. However, if the quality scalability is using MGS, switching different MGS layer can be done in any access unit. While CGS provides quality scalability by dropping complete enhancement layers, MGS provides a better granularity level of quality scalability by partitioning a given enhancement layer into several MGS layers. Individual MGS layers can then be dropped for quality (and bit rate) adaptation. With the MGS concept, any enhancement layer NAL (Network Abstraction Layer) unit can be discarded from a quality scalable bit stream.

\subsection{Network simulator II (NS-2) for evaluation transmission video}

The difficulties on research in video mainly arise from lack of tools and testing system environment (test bed) which can be accommodated by using network simulator. This paper describes about how to analyze video transmission using testbed on Network Simulator II (Version 2), widely known as NS-2 [18]. It is an event-driven tool which is useful for studying the dynamics of communication network. This test bed has simplicity in supporting researches on video transmission field [19]. NS-2 is very helpful for researchers in developing their works on many fields that constraint by laboratory tools or experiment related to networks. Video transmission over wireless network requires tools and complex technical environment. In this work, we have adopted and modified scenario networks in NS-2 (WLAN 802.11e) [20] from C. H. Ke [13].

\section{SYSTEM MODEL OF EVALUATION FRAMEWORK}

The proposed evaluation framework scheme is shown as in Figure 3 where it shows the simulation of bit rate; PSNR (Peak Signal-to-Noise Ratio); end-to-end delay; and queue length of packets. To analyze 
the outputs, we use MFC YUV viewer software and VLC media player. Source code of JSVM version 9.18 [21], runs on Linux Fedora 16. The main component of this framework evaluating as show in Figure 3 are as follows in Table 2. External tools from SVEF [11] adopted in this scheme as in Figure 2.

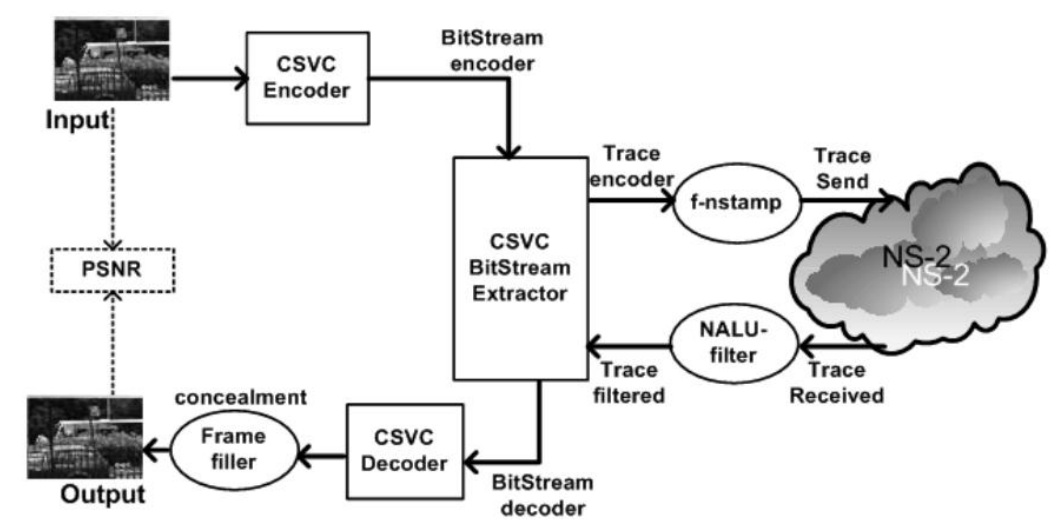

Figure 2. Novel approach of scheme for evaluating video transmission using CSVC with NS-2

\section{RESULTS AND ANALYSIS}

In this research, we propose a novel scheme, where CSVC is implemented over wireless broadband network WLAN IEEE 802.11e on NS-2 [17]. Figure 2 shows research scheme for evaluation framework overall. In this research, we use CGS and MGS modes of JSVM. In the experiment we use JSVM version 9.18 [21] and NS-2 version 2.29. Computer simulations are done to verify the performance of this new scheme. The parameters and components/tools of the scheme are given in Table 1.

Table 1. Parameters and Components/Tools in the evaluation frameworks

\begin{tabular}{cc}
\hline Parameters and Components/Tools & Description \\
\hline Input and output video sequences & Stefan (90 frames); \\
& Bus (150 frames) \\
& Foreman (300 frames); \\
& City (300 frames) \\
Encoder and Decoder od CSVC & Using JSVM version 9.18 [21] \\
BitStream Extractor CSVC & Part of JSVM \\
NS-2 & Version 2.29 [18] \\
SVEF & Version 1.4 and external tool [11] \\
PSNR Analyzer & External tool and optional \\
Spatial Scalable & QCIF; CIF \\
GOP (Group of Picture) & 4 frames \\
QP (Quantization Parameter) & $18 ; 20 ; 24 ; 32$ \\
Motion Search Range & \pm 32 \\
Wireless Standars & WLAN 802.11e [20] \\
Data rate & 1 Mbps \\
OS (Operating Systems) & Linux Fedora 16 \\
\hline
\end{tabular}

\subsection{Video quality parameters}

Testing and analysis of video input are Stefan sequences, Bus sequences, Foreman sequences, and City sequences. We use the Peak Signal-to-Noise Ratio (PSNR) to measure the quality between original sequence and reconstructed sequence. This metric employed is the Mean Squared Error (MSE) given by

$$
M S E=\frac{1}{W \cdot H} \sum_{x=0}^{W-1} \sum_{y=0}^{H-1}|f(x, y)-g(x, y)|^{2},
$$

and PSNR is defined as

$$
P S N R=10 \log _{10} \frac{\left(2^{n}-1\right)^{2}}{M S E}
$$


where $W$ is number of pixel/row, $H$ is number of row/frame, $f(x ; y)$ is luminance intensity of pixel in the original frame, $g(x ; y)$ is luminance intensity of pixel in the reconstructed frame, and $n$ is number of bits per pixel. In analyzing PSNR, we use the parameters of Y-PSNR (component of the video luminance) that are commonly used in the analysis of video transmission. Calculation and analysis of bit rate uses equation is given by

$$
B_{r}=\frac{N_{b}}{N_{f}} M_{f}
$$

where $\mathrm{Br}$ is bit rate, $\mathrm{Nb}$ is total bit, $N f$ is number of frame, and $M f$ is mean frame rate. Input video sequence has been analyzed by quantization parameters (QP) 20, 24, 28, and 32. In the calculation and analysis of endto-end delay and queue system, used equations such as packet transmission time given by

$$
T_{\text {packet }}=\frac{S_{\text {packet }}}{B_{t r}}
$$

where Tpacket is packet transmission time, Spacket is packet size, and Btr is bandwidth transmission. The analysis of the total delay is including of the propagation delay on the network.

\subsection{Performance evaluation of framework}

The main parameters used to evaluate framework performance are described as follows:

\subsubsection{Bitrate and efficiency coding}

In this research, the coding efficiency of encoder CSVC from Stefan sequence, Bus sequences, Foreman sequences, and City sequences are analyzed. This functions as an input as given in Figure 3 . We follow JSVM algorithm to operate encoding. The decoding output of CSVC is given in Fig 4. It shows that on layer 3, MGS mode gains PSNR $4 \mathrm{~dB}$ above CGS mode. Furthermore, it can be seen that there is no significant difference. Whereas, layer 1 there is $2.4 \mathrm{~dB}$ to $3.1 \mathrm{~dB}$ differences. This study utilize two modes: CGS and MGS in which each mode uses three layers of CSVC. It can be seen from layer 3 that MGS mode gains Y-PSNR about $2.2 \mathrm{~dB}$ above CGS mode at bit rate $1700 \mathrm{kbps}$. On the other hand, at Y-PSNR $42 \mathrm{~dB}$, MGS mode has a gap about $500 \mathrm{kbps}$ above CGS mode. On layer 2, it can be seen that there is not significant different. Whereas, it can be seen from layer 1 that there is $2.6 \mathrm{~dB}$ to $3.2 \mathrm{~dB}$ differences. There is a significant gap between CGS and MGS as shown in layer 3. In the case of Stefan sequence (Figure 3(a)) and BUS sequence (Figure 3(b)) as input, starts from $1000 \mathrm{kbps}(1 \mathrm{Mbps})$ bitrate has shown the gap, where the gap is $2.2 \mathrm{~dB}$ on $3700 \mathrm{kbps}$ bitrate. In case of Foreman sequence (Figure 3(c)) and City sequence (Figure 3(d)) as input, from $800 \mathrm{kbps}$ bitrate shows the gap, up to about $2700 \mathrm{kbps}$ bitrate shows around $1.2 \mathrm{~dB}$ gap. In contrast to the layer 1 and layer 2, it is not seen significant gap between CGS and MGS, the Stefan sequence and the BUS sequence as input below $1 \mathrm{Mbps}$ while Foreman sequence and City sequence as input below $800 \mathrm{kbs}$. We briefly note that MGS overcome PSNR performance to CGS at the same bitrate. Increasing number of frame sequence to be transmitted will cause Y-PSNR to have decreasing gap. We briefly note that MGS overcome PSNR performance to CGS at the same bitrate. Increasing the frame sequence to be transmitted causes Y-PSNR gap decreased. 


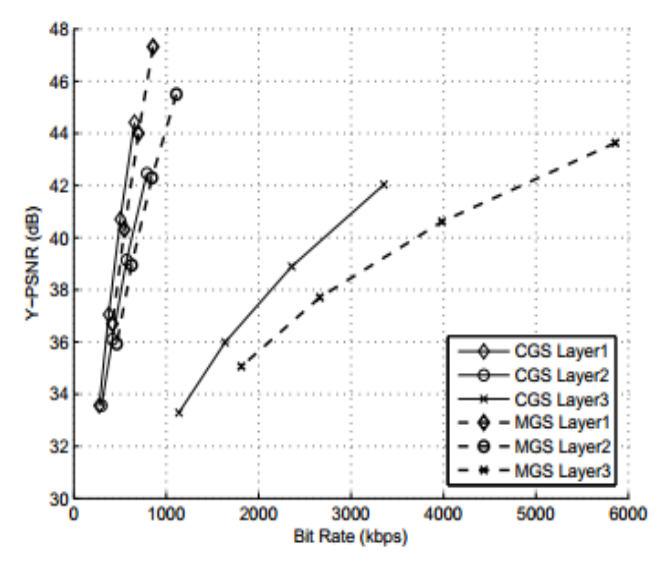

(a) Stefan sequence

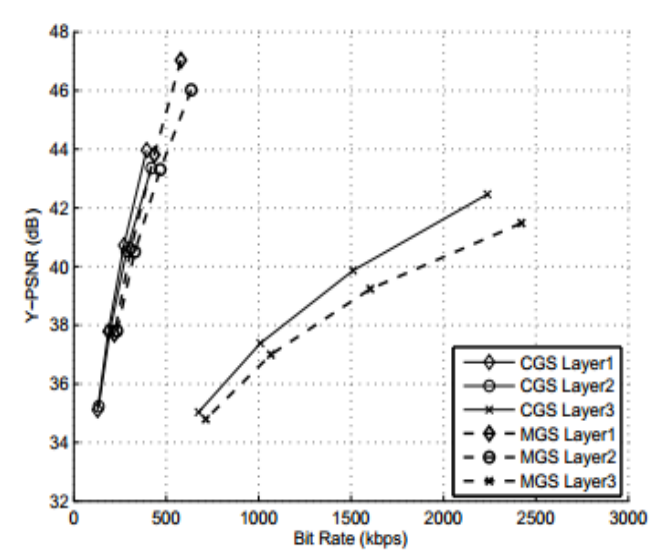

(c) Foreman sequence

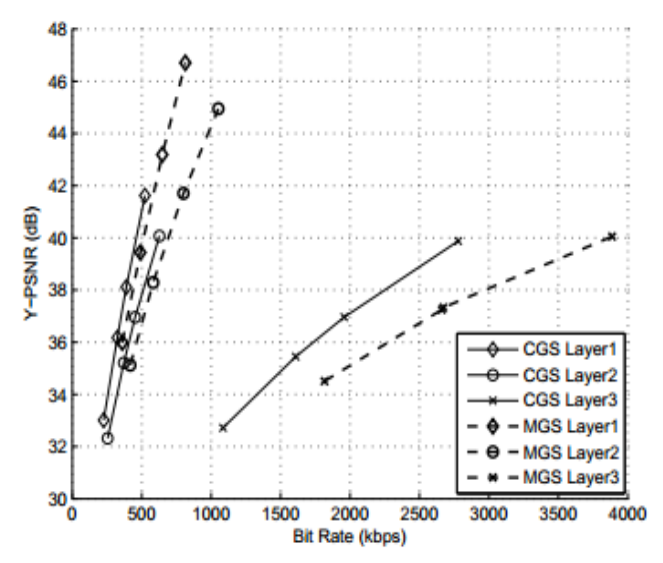

(b) Bus sequence

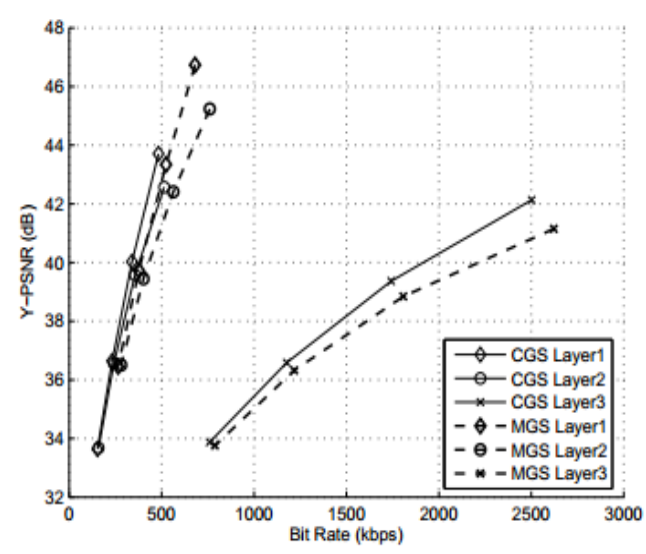

(d) City sequence

Figure 3. Performance of coding efficiency component of encoding

\subsubsection{PSNR as performance framework overall}

Figure 4 Stefan sequence shows that the average Y-PSNR as output of the new scheme, where CGS has value of $16.8 \mathrm{~dB}$, and MGS $20 \mathrm{~dB}$. It is around $3.3 \mathrm{~dB}$ gap. In Figure 4(b) on Bus sequence, where CGS has value of $11.9 \mathrm{~dB}$, and MGS $14.6 \mathrm{~dB}$. It has around $2.8 \mathrm{~dB}$ gap. In Figure 4(c) Foreman sequence, where CGS has value of $23.3 \mathrm{~dB}$, and MGS $25.7 \mathrm{~dB}$. It has around $2.45 \mathrm{~dB}$ gap. In Figure 4(d) City sequence, it shows that CGS has value of $23.5 \mathrm{~dB}$, and MGS has the value of $24.8 \mathrm{~dB}$. It has around $1.4 \mathrm{~dB}$ gap. We can conclude that MGS is better than CGS of approximately $1.4 \mathrm{~dB}$ up to $3.1 \mathrm{~dB}$. However from the ITU reference [22], it showed that the results mentioned above still exist under the fair category (PSNR below $25 \mathrm{~dB}$ ). The only worth more than $25 \mathrm{~dB}$ is MGS mode on the input of Foreman sequence. This shows that the error-resilience and the error-concealment has not running well where packet/frame error is still a lot going on. NS-2 environment affects the results, so that the development it is a challenge for the future research. 


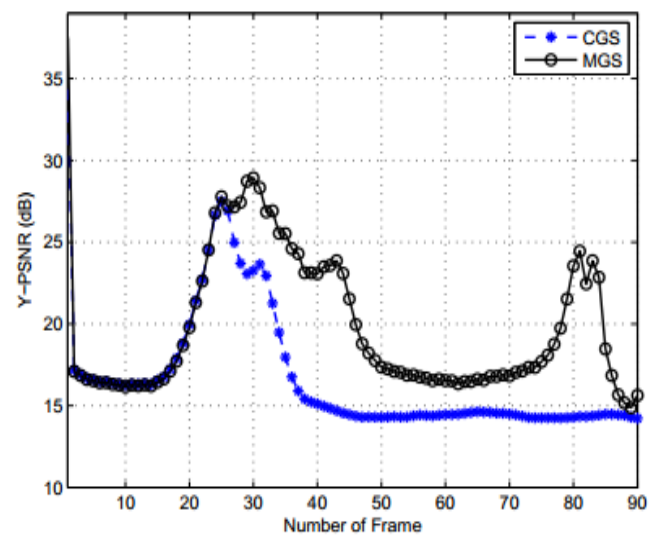

(a) Stefan sequence

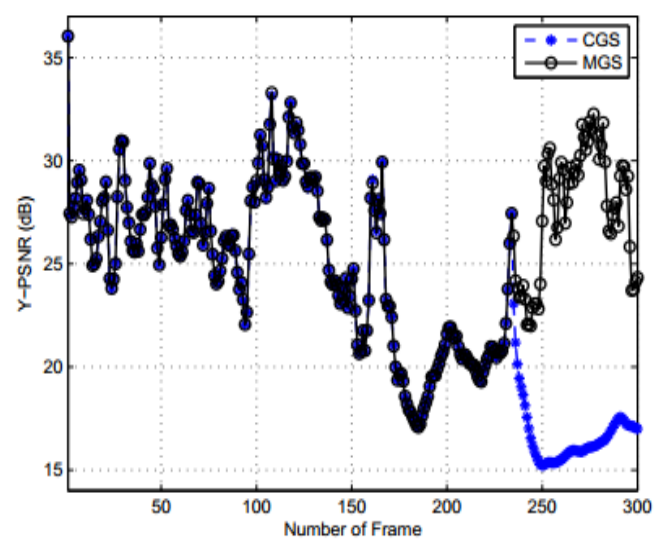

(c) Foreman sequence

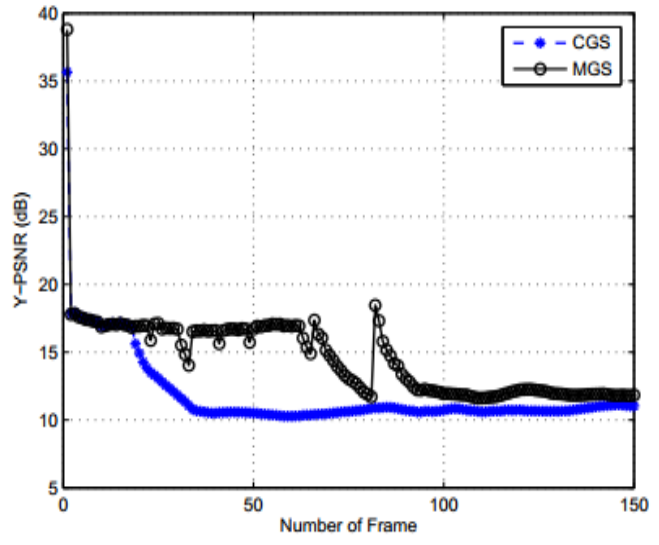

(b) Bus sequence

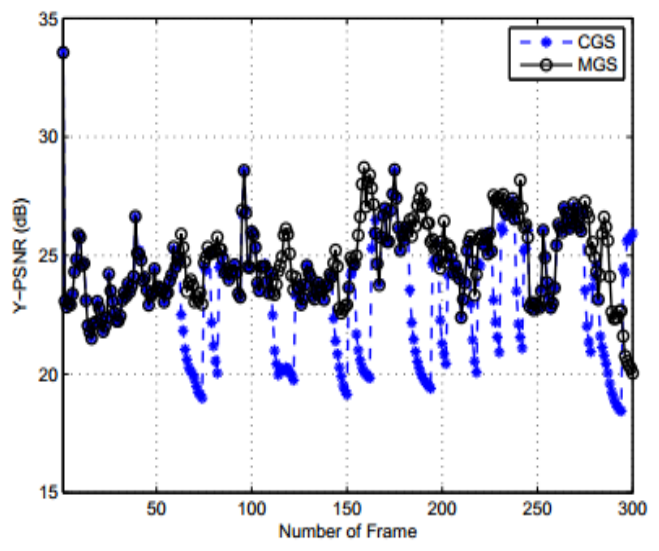

(d) City sequence

Figure 4. Performance of PSNR metrics of the proposed scheme

\subsubsection{End-to-end delay}

Parameter of end-to-end delay depends on the characteristics of the input sequence to be transmitted. CGS is relatively more stable as input Stefan sequence (as show Figure 5(a)) and the Bus sequence (as show Figure 5(b)). It shows that the packet delivery is delayed to 100 packets in 0.8 seconds, while in MGS, end-to-end delays is increasing in linear. On the input of Foreman sequence (as show Figure 5(c)) and the City sequence (as show Figure 5(d)), CGS has an average delay of about 0.7 seconds, while MGS has an average delay of about 0.2 seconds. It indicates that MGS has less end-to-end delay and better than CGS. The more packets transmitted in the network, the gap of delay occurs between CGS and MGS is higher.

\subsubsection{Queue length systems}

In this study we limit the system to the maximum packet queue of 50 packets in the buffer due to the computational efficiency issues. We observe from Figure 6 that the Foreman sequence (Figure 6(a)) and City sequence (Figure 6(b)) show that CGS has less time than MGS. However, in Foreman sequence (Figure 6(c)) and City sequence (Figure 6(d)) as the inputs which have more number of frames show that queuing length of MGS is less than CGS. Increasing number of frames sequence in transmission will cause queuing of packets takes longer time. In summary, we conclude that CGS and MGS mode on video transmission over wireless broadband network can be implemented on NS-2 well. The use of MGS mode is more satisfactory compared to CGS mode. 


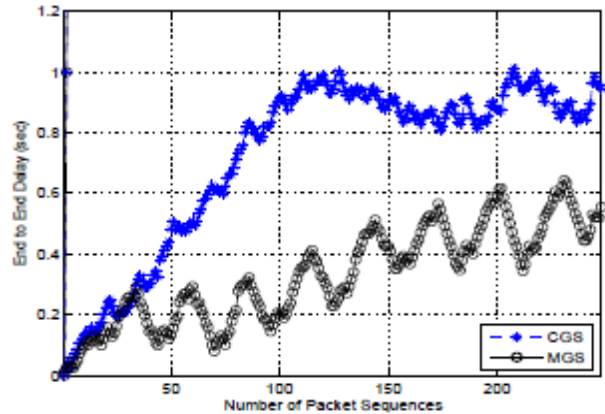

(a) Stefan sequence

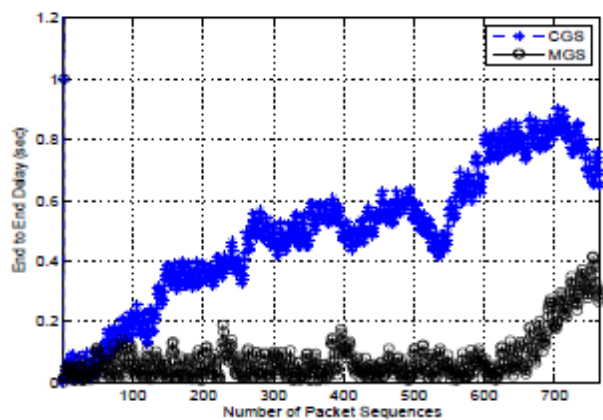

(c) Foreman sequence

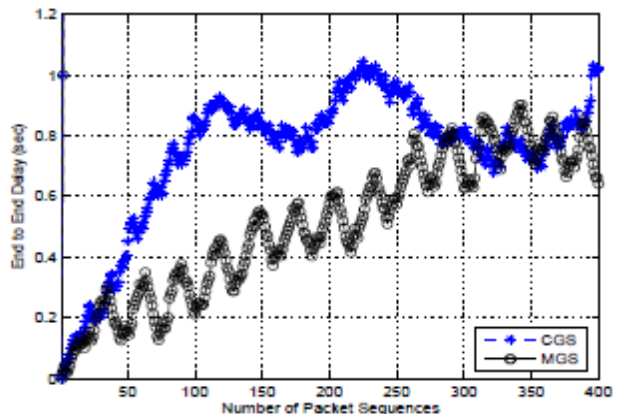

(b) Bus sequence

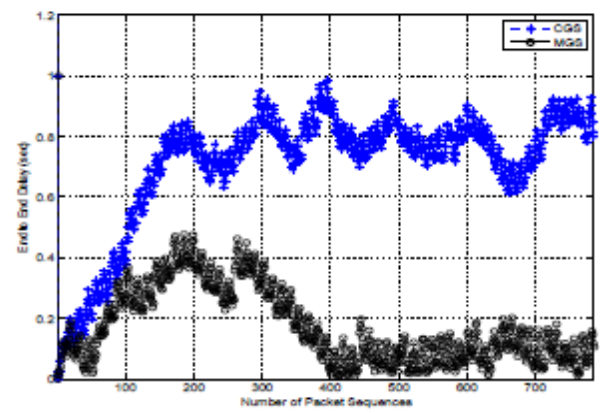

(d) City sequence

Figure 5. Performance metrics systems on the end-to-end delay

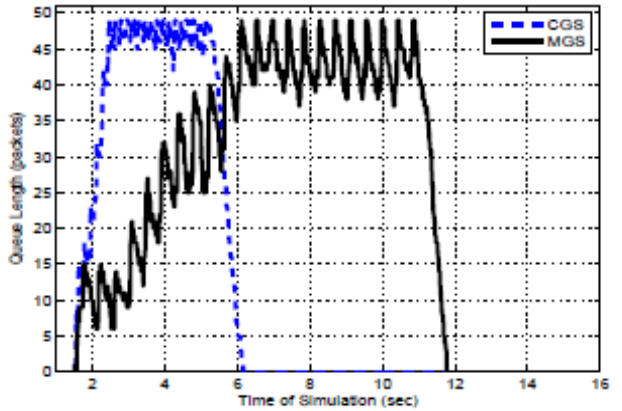

(a) Stefan sequence

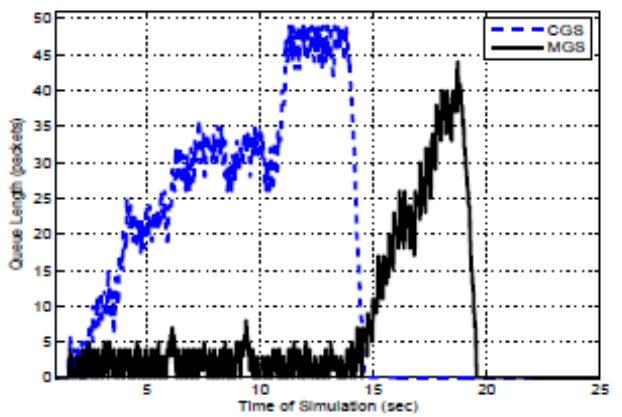

(c) Foreman sequence

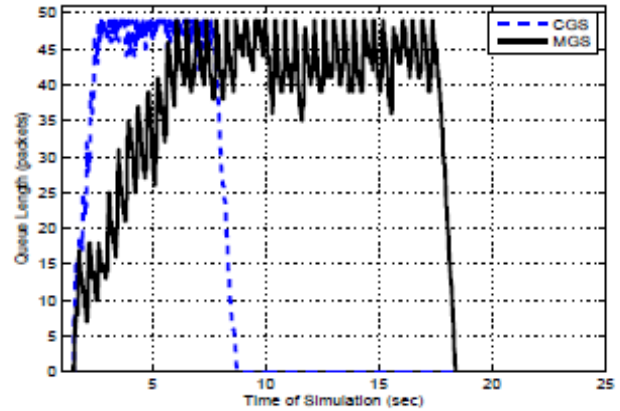

(b) Bus sequence

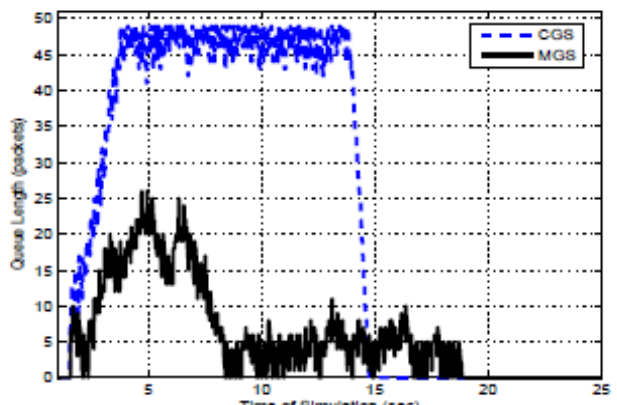

(d) City sequence

Figure 6. Performance metrics systems on the queue length packets 


\section{CONCLUSION}

In this research, we have proposed a novel approach for platform evaluation of video transmission based on the CSVC over wireless broadband network (WLAN IEEE 802.11e), simulated by NS-2. Furthermore, we also have investigated the effects of the application of MGS and CGS modes on performance of this system. The application of MGS mode on CSVC increases the performance of the system in comparison to CGS mode. In general, our scheme is simple and easily implemented for evaluating video transmission over wireless broadband network.

The future work will focus on providing CSI (Channel State Information) using limited feedback method for new scheme on adaptive system for video transmission over wireless broadband network services such WiMAX or LTE. Moreover, we will extend and develop our future results by implementing NS-3 and then compare the results to the real experiment.

\section{ACKNOWLEDGEMENTS}

The research is supported through Post-Doctoral Grants (Hibah Pascadoktor) by Indonesian Ministry of Research and Higher Education (under Grant No. 223/UN15.19/LT/2017). We are very grateful to Mr. Chih-Heng Ke for discussion and development of NS-2 simulation program; Prof Hua An-Zhao for support; Lab. Teknik Elektro FST Undana; Communication Digital Lab. UI; Mr. Dewa Made Wiharta and Mr. Meksianis Ndii for fruitful discussion.

\section{REFERENCES}

[1] Wu, Y. T. Hou, and Y.-Q. Zhang, "Scalable Video Coding and Transport over Broadband Wireless Networks", Proceeding of the IEEE, vol. 89, no. 1, pp. 6-20, 2001.

[2] H. Schwarz, D. Marve, T. Wiegand, "Overview of the Scalable Video Coding Extension of the H.264/AVC Standard", IEEE Transaction on Circuits and Systems for Video Technology, vol. 17, no. 9, pp. 1103-1120, 2007.

[3] M. Wien, H. Schwarz, and T. Oelbaum, "Performance Analysis of SVC", IEEE Transaction on Circuits and Systems for Video Technology, vol. 17, no. 9, pp. 1194-1203, 2007.

[4] T. Schierl and T. Wiegand, "Mobile Video Transmission using SVC", IEEE Trans. Circuits Syst. Video Technol, vol. 17, no. 9, pp. 1204-1217, 2007.

[5] M. van der Schaar and H. Radha, "A Hybrid Temporal-SNR Fine-Granular Scalability for Internet Video", IEEE Transactions on Circuits and Syst. for Video Technol., vol. 11, no. 3, 2001.

[6] L. Baszak, M. Domaski, and S. Makowiak, "Spatio-Temporal Scalability in AVC Codec", In: ISO/IEC JTC1/SC29/WG11, MPEG2003/M9469, Pattaya, Thailand, 2003.

[7] H. Schwarz, D. Marve, T. Schierl, and T. Wiegand, "Combined Scalability Support for the Scalable Extension of H.264/AVC", In Proc. IEEE Int. Conference on Multimedia and Expo, 2005.

[8] M. Li, Z. Chen, and Y. Tan, "Cross-layer Optimization for SVC Video Delivery over the IEEE 802.11e Wireless Networks", Journal of Visual Communication and Image Representation, vol. 22, no. 3, pp. 284-296, 2011.

[9] J. Klaue, B. Rathke, and A. Wolisz, "Evalvid A Framework for Video Transmission and Quality Evaluation", Computer Performance Evaluation. Modelling Techniques and Tools, Lecture notes in Computer Science, pp. 255-272, 2003.

[10] C. H. Ke, C. K. Shieh, W. S. Hwang, and A. Ziviani, "An Evaluation Framework for more Realistic Simulations of MPEG Video Transmission”, Journal of Information Science and Engineering, vol. 24, no. 2, pp. 425-440, 2008.

[11] A. Detti, et al., "SVEF: An Open-Source Experimental Evaluation Framework for H.264 Scalable Video Streaming", In Proceeding of IEEE MediaWIN, Sousse, Tunisia, 2009. [Online]. Available: http://svef.netgroup.uniroma2.it/

[12] T. A. Le and H. Nguyen, "End-to-End Transmission of Scalable Video Contents: Performance Evaluation over EvalSVC $\square$ a New Open-Source Evaluation Platform”, Journal Multimedia Tools and Applications, Springer, vol. 63, April 2013. [Online]. Available: http://evalsvc.sourceforge.net/

[13] C. H. Ke, "myEvalSVC: an Integrated Simulation Framework for Evaluation of H.264/SVC Transmission", KSII Trans. on Internet and Information Systems, vol. 6, no. 1, pp. 378-393, January 2012. [Online].Available: http://140.116.72.80/ smallko/ns2/svc.htm

[14] K. Rantelobo, Framework and Tool-Set for Evaluating Video Transmission Using CSVC, [Online].Available: $\mathrm{http}: / /$ sites.google.com/site/kalveinrantelobo/csvc.

[15] K. Rantelobo, K. A. Nugraha, Wirawan, and G. Hendrantoro, "Combined Scalability Technique for Video Transmission over Wireless Channel with MIMO-OFDM Systems", In Proceeding of International Conference on Instrumentation, Communication, Information Technology, and Biomedical Engineering (ICICI-BME) 2009, ITB, Bandung, Indonesia, 2009.

[16] K. Rantelobo, Wirawan, G. Hendrantoro, A. Affandi, and H. Zhao, "Adaptive Combined Scalable Video Coding over MIMO-OFDM Systems using Partial Channel State Information”, KSII Trans. on Internet and Information Systems, vol. 7, no. 12, pp. 3200-3219, Dec. 2013.

[17] P. Seeling, M. Reisslein, and B. Kulapala, "Network Performance Evaluation using Frame Size and Quality Traces of Single-Layer and Two-Layer Video: A Tutorial", IEEE Communications Surveys Tutorials, vol. 6, no. 3, pp. 58-78, 2004. 
[18] Network Simulator II (NS-2), [Online]. Available: http://www.isi.edu/nsnam/ns

[19] T. Issriyakul and E. Hossain, Introduction to Network Simulator NS2, Springer, NY, 2009.

[20] IEEE Standard 802.11e, "Wireless LAN Medium Access Control (MAC) and Physical Layer (PHY) Specification. Amendment 8: MAC Quality of Service Enhancement", 2005

[21] ITU-T and ISO/IEC (JVT): Joint Scalable Video Model JSVM version 9.19. March, 2011. [Online].Available: http://ftp3.itu.int/av-arch/jvt-site

[22] ITU-T, ITU Recommendation g.1010: End-User Multimedia QoS Categories, 2001.

\section{BIOGRAPHIES OF AUTHORS}

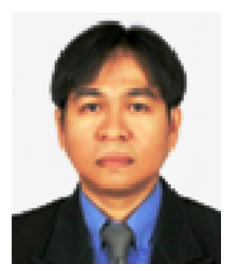

Kalvein Rantelobo received the B.E. degree from Hasanuddin University, Makassar, Indonesia in 1996; M. Eng. degree from Institut Teknologi Bandung (ITB), Bandung, Indonesia in 2003; and the Dr. Eng. degree from Electrical Engineering Dept. at Institut Teknologi Sepuluh Nopember (ITS), Surabaya, Indonesia in 2014. Since 1998 he has been with Electrical Engineering Department Universitas Nusa Cendana as lecturer. His research areas include video transmission over mobile broadband services, video coding and Multimedia Wireless Sensor Networks. He is a member of IEEE.

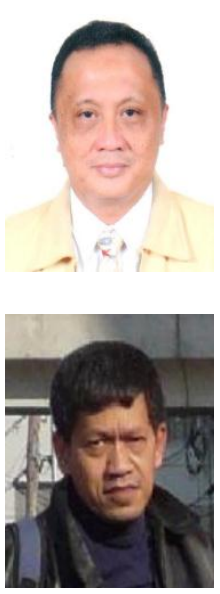

Kalamullah Ramli is a Professor on Computer Engineering since July 1, 2009. He finished his Masters in Telecommunication Engineering at University of Wollongong, NSW, Australia, in 1997. He then continued his Doktorarbeit on Computer Networks in year 2000 at Universitaet Duisburg-Essen, NRW, Germany, and obtained his Dr.-Ing. in year 2003. He has been with Universitas Indonesia (UI) since 1994. His research interests are Pervasive and Embedded System; Intelligent Transportation System; Network Modeling and Protocol Management; and Strategic ICT Policy and Regulation.

Wirawan received the B.E. degree in telecommunication engineering from Institut Teknologi Sepuluh Nopember (ITS), Surabaya, Indonesia, in 1987, the DEA in signal and image processing from Ecole Supérieure en Sciences Informatiques, Sophia Antipolis, and the Dr. degree in image processing from Telecom ParisTech (previously Ecole Nationale Supérieure des Télécommunications), Paris, France, in 1996 and 2003, respectively. Since 1989 he has been with ITS as lecturer in the Electrical Engineering Department. His research interest lie in the general area of image and video signal processing for mobile communications, and recently focuses more on underwater acoustic communication and networking and on various aspects of wireless sensor networks. He is a member of IEEE 